\title{
La interculturalidad en un aula de primaria: tensiones durante el tratamiento de la categoría inmigrante
}

- Emilio Tevez y Sofía Dueñas Díaz

\begin{abstract}
Resumen
Este artículo problematiza la construcción sociocultural de la categoría inmigrante en el aula escolar. A partir de ello se establecen dos objetivos. Por un lado, visualizar cómo la construcción social de la categoría inmigrante está atravesada por formas identitarias establecidas por formaciones nacionales de alteridad hegemónicas. Por el otro, dar cuenta de cómo a dichas formaciones se contraponen construcciones de un "otro" producidas por los actores sociales en su experiencia cotidiana. El abordaje se realizó mediante el enfoque etnográfico en tanto permite el relevamiento de situaciones cotidianas. La principal estrategia de investigación fue la observación participante, puntualizando el registro en la interacción de la docente y los y las estudiantes en el tratamiento del tema inmigración en una escuela primaria privada a la que concurren inmigrantes de países limítrofes en la ciudad de Olavarría (provincia de Buenos Aires, Argentina). La investigación permitió reconocer la complejidad con la que se constituyen las definiciones hegemónicas de inmigrante -que incluyen tanto aspectos objetivos como subjetivos- en las prácticas desarrolladas por los actores. Estas construcciones se establecieron a partir del ideario sobre el "ser nacional" que invisibiliza procesos sociales que hacen a la existencia de múltiples "otredades". Asimismo, mediante el registro de la actividad escolar, se identificaron otros procesos que desnaturalizaron dichas definiciones hegemónicas.
\end{abstract}

\section{Interculturality in a primary classroom: tensions during treatment of the immigrant category}

\begin{abstract}
This article problematizes the sociocultural construction of the immigrant category in a school classroom. For this, two objectives are established. On the one hand, visualizing how the social construction of the migrant category is crossed by identitary forms
\end{abstract}

\section{Palabras clave:}

Inmigrante, identidad, alteridad, escuela, frontera.

\section{Keywords:}

Migrant, identity, otherness, school, border. 
established by national formations of hegemonic alterity. On the other, give an account of how such formations are opposed constructions of an "other" produced by social actors in their daily experience. The approach was carried out through an ethnographic approach in order to survey everyday situations. The main research strategy was participant observation pointing out the record in the interaction of the teacher and the students in the treatment of the migration issue in a private primary school attended by migrants from neighboring countries located in the city of Olavarría (province of Buenos Aires, Argentina). The investigation allows to recognize the complexity in which the hegemonic definitions of migrant - which include both objective and subjective aspects- are constituted in the practices developed by the actors. These constructions are based on the ideology of the "national being" that invisibilizes social processes that make the existence of multiple "othernesses". Also, by registering the school activity other processes that denature these hegemonic definitions are identified.

\section{Introducción}

El artículo forma parte de una experiencia de campo realizada entre 2011 y 2015 , constituyendo uno de los primeros antecedentes para la conformación del Grupo de Investigaciones Socioculturales en Educación (GISE) que tiene lugar en la Facultad de Ciencias Sociales (UNICEN). En la actualidad, las actividades que se desarrollan tienen como eje el proyecto: Diversidad cultural y formación docente: un estudio sobre la incidencia de la interculturalidad en las experiencias formativas de estudiantes de profesorado de educación primaria en una localidad de la Provincia de Buenos Aires. Allí se propone abordar el enfoque sobre la diversidad cultural producido en la región con especial atención al tratamiento de pueblos originarios, población migratoria, la perspectiva de género y la discapacidad. Todo esto, identificando las particularidades que adquiere la perspectiva intercultural en el sistema escolar del centro de la Provincia de Buenos Aires. La propuesta del presente artículo tuvo sus inicios abordando las maneras en que niñas/os y sus docentes de nivel primario construían la categoría inmigrante en el contexto escolar. Sobre ello, interesa indagar y reflexionar la forma en que la categoría inmigrante es atravesada por aspectos identitarios establecidos por formaciones nacionales de alteridad de carácter hegemónico y cómo se contraponen a dichas formaciones las construcciones de un "otro" inmigrante producidas por los actores -principalmente las/os niñas/os- en su experiencia cotidiana. El interés por abordar dicha problemática se vincula a lo que Feldman-Bianco, Rivera Sánchez, Stefoni y Villa Martínez (2010) plantean como uno de los temas centrales vinculado a los inmigrantes. Esto es, la producción de fronteras geopolíticas y simbólicas a las que dicha población se enfrenta en situaciones que experimentan cotidianamente y que van más allá de los límites territoriales entre países. Para comprender cómo operan estas nociones, a lo largo del trabajo se hace referencia al registro de dos clases en una escuela primaria en las que se abordó el tema y se identificaron situaciones donde, al nombrarse al inmigrante, se establecieron diferenciaciones entre "ellos" (inmigrantes) y "nosotros" (argentinos/as). En los registros de las clases se identificaron "... ciertos dispositivos categoriales que, a la vez que nombran, clasifican y otorgan sentido, separan mundos, crean fronteras, construyen, incorporando o excluyendo tipos diferenciados de sujetos inmigrantes" (Feldman-Bianco, Rivera Sánchez, Stefoni \& Villa Martínez, 2010, p. 15).

Considerando lo anterior, el supuesto que moviliza la realización de este trabajo plantea que la construcción social de la categoría inmigrante está atravesada por formas identitarias que los actores sociales producen y reproducen. Las mismas son condicionadas por formaciones nacionales de alteridad (Briones, 2005; Segato, 2007) que, al mismo tiempo, son fragmentadas por los mismos actores a partir de sus experiencias cotidianas, existiendo maneras alternativas de construir al inmigrante más allá de las fronteras nacionales. 
El lugar en el que se realizó el trabajo de campo fue la "Escuela Franciscana", una institución católica de gestión privada inaugurada en 1960 y reconocida en la región por su histórica vinculación con poblaciones de inmigrantes. Está ubicada en la región sudoeste de la ciudad y desde su fundación concurren niños y niñas de familias provenientes de Bolivia. Al momento de realizar la investigación, la población inmigrante había crecido en heterogeneidad identificándose estudiantes de Chile, Paraguay y República Dominicana. A continuación, el trabajo se divide en cuatro apartados. En el primero se hace referencia a los diferentes enfoques que tratan lo planteado en el párrafo anterior y se explicitan las categorías seleccionadas para analizar lo relevado durante la estadía en la escuela. Además, se plantean algunas características del contexto en el que se realizó el trabajo de campo y algunos señalamientos teórico-metodológicos enfatizando la relevancia y aportes del enfoque etnográfico para el tratamiento del tema (Rockwell, 2009). El segundo apartado refiere a las características que asume la construcción social del inmigrante por parte de la docente y el texto escolar con el que propone los contenidos y la consigna del tema. Particularmente, se puntualiza en la incidencia que genera en ese proceso la construcción del "ser nacional" a partir de las formaciones nacionales de alteridad y cómo estas se expresan en el contexto del aula. El tercer apartado puntualiza en las fracturas que sufre la construcción de la categoría inmigrante que realiza la docente mediante los discursos de los niños y una inmigrante invitada para hablar de su país y experiencia. De esta forma, se identifican aspectos vinculados a las formaciones interculturales al interior del territorio nacional y a la existencia de campos sociales transnacionales que se oponen a las construcciones hegemónicas. En este proceso, se ponen de relieve las tensiones que se producen entre las construcciones sobre el inmigrante producidas a partir de las interacciones que establecen los actores. Finalmente, en el último apartado, se desarrollan algunas reflexiones sobre el trabajo realizado con énfasis en los interrogantes que genera este tipo de abordaje.

\section{Aspectos teórico-metodológicos}

El artículo se inscribe en el campo subdisciplinar de la Antropología y Educación. Particularmente, en lo que refiere al estudio de las relaciones interculturales en contextos escolares. En Argentina fue en la década de 1990 -en un contexto de profunda desigualdad social- que la relación entre la escuela y poblaciones definidas como otredades se constituyó como tema de interés. Al tratamiento del tema mediante categorías como multiculturalidad o diversidad, se incorporó el reconocimiento de las relaciones de subalternización en contextos de pobreza (Neufeld, 2009). Sobre dicho recorrido se ha elaborado cuantioso material que se constituye en antecedentes ya que problematizan la presencia de otredades en las escuelas (Franzé Mudanó, 2002; Neufeld y Thisted, 1999; Novaro, 2011a; Sinisi, 1999). En este caso, con el objetivo de comprender el relevamiento realizado sobre la construcción de la categoría inmigrante, resulta necesario hacer referencia a los procesos que constituyen a los grupos sociales. En principio, reconocer que es a partir de las fronteras intergrupales donde se establecen las configuraciones que producen los criterios y entrenamientos que definen a la persona entendida (Levinson \& Holland, 1996) -o integrante del grupo-. Como plantea Barth (1976), las fronteras deben ser entendidas como formas de organización que se producen y reproducen mediante un conjunto de adscripciones categoriales que clasifican a las personas de acuerdo a una identidad básica y general. Las personas utilizan esas adscripciones para definir un "nosotros" y un "otro", estableciéndose a partir de esta interacción las definiciones identitarias de un determinado grupo social. Es el límite entre el "nosotros" y el "otro" lo que define al grupo y no el contenido cultural que lo encierra. Por ello, los rasgos que lo caracterizan no constituyen diferenciaciones objetivas, sino que son seleccionados a partir de lo que los actores consideran significativo. Esa identificación como miembros del mismo grupo que es arbitraria y socialmente construida, entraña
1. A fin de mantener el anonimato de la población de estudio se han modificado los nombres de las personas y lugares para resguardar sus identidades. 
una coparticipación de criterios de valoración y de juicio en el que estos se encuentran "jugando al mismo juego" (Barth, 1976). La dicotomía que convierte a "otros" en extraños -miembros de otro grupo- supone un reconocimiento de las limitaciones para llegar a un entendimiento recíproco. Los límites se expresan en las diferencias de criterios para emitir juicios de valor o conducta, restringiendo de la interacción a posibles sectores que presuponen común acuerdo e interés. Esos límites, que siempre son sociales, tienen una expresión territorial.

Las fronteras asumen diversas formas. Una de ellas, delimita la soberanía nacional mantenida y administrada por cada Estado. Allí se articulan sistemas económicos, estructuras sociales, instituciones jurídicas y políticas y aparatos ideológicos que van "entramando" formaciones nacionales de alteridad. Estas últimas “... no solo producen categorías y criterios de identificación/clasificación y pertenencia, sino que regulan condiciones de existencia diferenciales para los distintos tipos de otros internos que se reconocen como formando parte histórica o reciente de la sociedad sobre la cual un determinado Estado nación extiende su soberanía" (Briones, 2005, p. 16). Los ejes que atraviesan estas formaciones asumen características que le dan contenido a las categorías sociales que hacen a esas configuraciones, así como esas categorías le dan sentido a aquellas fuerzas económicas, políticas y sociales. Como plantea Briones (2005), este doble proceso es un componente irreductible de las identidades colectivas. Para comprender cómo se han constituido, es necesario reconocer el establecimiento de las alteridades formadas a lo largo de las historias nacionales (Segato, 2007). La autora plantea que dichas alteridades son resultado de fronteras históricas interiores, con origen en el mundo colonial y continuidad en el contexto demarcado por los estados nacionales. La escuela es uno de los espacios donde se estructuran estas fronteras (Briones, 2005).

$\mathrm{Al}$ abordar la forma en que se constituyen las formaciones nacionales de alteridad, es necesario reflexionar sobre los procesos que hicieron posible su desarrollo. En principio, se debe tener presente que no es lo mismo objetivar dichas formaciones que naturalizarlas, entendiendo que la relación entre Estado nación y sociedad no es algo dado (Levit \& Glick Schiller, 2004). Teniendo en cuenta ello es importante considerar dos cuestiones. Por un lado, que las formaciones identitarias son construcciones producidas a partir de tensiones y conflictos existentes entre diversos grupos sociales. Por el otro, que el establecimiento de un territorio vinculado a formaciones identitarias es producto de una historia en la que se imponen determinados sectores -dominantes- sobre otros. Sin embargo, a pesar de su fuerza, existen fracturas en ese poder que se producen mediante las acciones de sectores subalternos que resiste y se oponen. Para comprender este proceso, es pertinente la categoría hegemonía que constituye, como plantea Williams (1977) a partir del trabajo de Gramsci, un complejo entrelazamiento de fuerzas políticas, sociales y culturales. Su incorporación tiene la intención de evitar análisis mecanicistas que conciben a los actores como simples reproductores de ideología dominante, dando cuenta de la complejidad con que se produce la dinámica social de los grupos.

La forma en que se establece la hegemonía se produce a partir de un cuerpo de prácticas que abarcan la totalidad de la vida, incluyendo las percepciones que los actores tienen de ellos mismos. Estas, en tanto significados y valores que son experimentados por la población, son confirmadas recíprocamente. De esta manera, se constituyen en un sentido de la realidad para la mayoría de la gente en una sociedad determinada (Williams, 1977), lo que implica una cultura que debe ser considerada como la vívida dominación y subordinación de clases particulares. Sin embargo, la hegemonía no es total ni eterna, sino que es continuamente resistida, limitada, alterada y desafiada por formas alternativas o directamente opuestas, expresadas en las prácticas de los dominados. A partir de estas consideraciones, se abordarán las diversas formas que asume la construcción social del inmigrante en el contexto escolar, visualizando orientadores que permiten analizar el registro de campo y la complejidad con la que se expresa el proceso. 
El abordaje metodológico se produjo mediante el enfoque etnográfico, permitiendo el relevamiento de situaciones cotidianas y el registro de la forma en que las personas producen y reproducen prácticas y representaciones. De esta forma, se implementaron estrategias de investigación que permitieron documentar lo no documentado (Rockwell, 2009) a partir de la interacción con la población de estudio entre los años 2011 y 2015. La principal estrategia de investigación fue la observación de situaciones de aula, puntualizando el registro en la interacción de la docente y los/las estudiantes en el tratamiento del tema inmigración. En este sentido, la etnografía permitió “... documentar en el tiempo cómo las personas, simultáneamente, mantienen y modifican repertorios e identidades culturales, interactúan dentro de una localidad y más allá de sus fronteras; además de actuar de modos que son congruentes o contradicen sus valores" (Levit \& Glick Schiller, 2004, p. 70).

El contexto en el que se realizó la investigación fue la ciudad de Olavarría, fundada el 25 de noviembre de 1867 y ubicada en el centro de la provincia de Buenos Aires a 350 kilómetros de la Ciudad Autónoma de Buenos Aires. Allí se encontraban las instituciones que administraban el partido, lo que la convertía en la ciudad cabecera de doce localidades que iban de poblados con centros fabriles a parajes rurales. Su denominación de rango medio refería a la interrelación entre lo rural y lo urbano que se expresaba en sus principales actividades económicas: la explotación agroganadera y la industria minera. En ese momento, según los datos del Censo Nacional de Población, Hogares y Viviendas 2010 (Indec), la población del partido era de 111.058 habitantes. Sobre ese total 1.650 habitantes ingresaban en la categoría de extranjero constituyendo el 1,5\% de la población que la ubicaba por debajo de la media nacional $-4,5 \%$ siendo un total de 1.805.957 migrantes- (El Popular, 31 de marzo de 2014). La procedencia de los grupos de migrantes que llegaban a la ciudad se había diversificado. Del total del $1,5 \%$ la mayoría $(63,5 \%)$, pertenece al continente americano, siendo el $90 \%$ de países limítrofes mayoritariamente de Paraguay, Chile y Bolivia.

Al sudoeste de la ciudad se ubicaba la "Escuela Franciscana", lugar en el que se realizó el trabajo de campo, en un contexto conocido como "el barrio de los bolivianos".2 Allí se encontraba la sede de la Asociación de Residentes Bolivianos de Olavarría (ARBO) y tenían domicilio los primeros migrantes que llegaron de ese país. Según el informe titulado Diagnóstico Educativo -elaborado desde la escuela-, en los barrios que residía los alumnos de la escuela existían escasos estímulos y recursos materiales, con excepción de los Servicios Municipales Territoriales. El diagnóstico mencionado, haciendo referencia a ese momento, planteaba: otro hecho trascendente para la comunidad fue la llegada a la zona de varias familias bolivianas (a mediados de 1950), atraídas por la oferta laboral de las fábricas cementeras, que vinieron en búsqueda de mejores condiciones de vida. La historia de más de medio siglo con los inmigrantes le dio a la institución una impronta particular. La escuela generó iniciativas como la elaboración de un "Proyecto Intercultural" orientado a familias bolivianas, al identificar problemáticas vinculadas a la diferencia del lenguaje, analfabetismo, cuestiones de género -centrado en el machismo de los hombres bolivianos-, entre otros.

La vinculación entre la escuela y poblaciones migrantes era reconocida también por las familias. Durante una entrevista una mujer comparaba la experiencia que habían tenido sus hijos en otras instituciones educativas, donde docentes y estudiantes los discriminaban por ser extranjeros. Esta situación motivó que la mujer decida sacarlos de allí e incorporarlos a la escuela "Franciscana", donde permitían abanderados extranjeros y participaban en actividades extraescolares como la celebración de la Virgen de Copacabana. En relación a la Ley Migratoria 25871, la escuela tenía presente el derecho de ingreso de migrantes cualquiera fuese su situación administrativa. Para resolver temporalmente el inconveniente, solían evaluar al estudiante para identificar "lo que sabía" y ubicarlo en el grado correspondiente. El mecanismo reemplazaba el
2. Sobre esta apreciación se destaca la respuesta de entrevistados que al referirse a ese lugar lo ubicaban como la parte "trasera" de la ciudad: “'¿La parte de atrás? Es adonde uno pondría la basura: los barrios donde viven los bolivianos...', mientras que otra persona consideraba que '... el barrio de bolivianos es como de indios, no es Del Centro eso..."' (Gravano, 2006, p. 318). 
boletín de calificaciones permitiendo a la persona iniciar los estudios, cumpliendo con el objetivo de inclusión que tenía la institución (Tevez, 2011). Particularmente, las observaciones se realizaron en tercer grado del nivel primario a donde concurrían una veintena de niñas/os de siete y ocho años, entre las/os que había inmigrantes provenientes de Bolivia y Chile. Sobre el abordaje realizado "... interesa plantear una discusión que supere el plano del estudio de las acciones individuales. La cuestión aquí, no es culpabilizar a docentes o a niños, sino intentar comprender que las tramas de significación construidas que definen y excluyen al 'diferente'...' (Sinisi, 1999, p. 190).

\section{La frontera nacional como distinción unívoca en la definición de inmigrante}

En Argentina durante el proceso de construcción nacional se “... presionó para que la nación se comportase como una unidad étnica dotada de una cultura singular propia y homogénea y reconocible" (Segato, 2007, p. 10). Como afirma la autora, la escuela es una de las instituciones que contribuyó para que la idea de nación se construyera, convirtiéndose en la gran antagonista de las minorías. En esta línea, Briones (2005) plantea que la base de este proceso se estableció sobre un ideario de nación homogénea, blanca y europea mediante la metáfora crisol de razas, con el objetivo de liberar la identificación nación de componentes étnicos particulares -principalmente, a los pueblos originarios-. Así, la idea de ser nacional -"nosotros"- se estableció a partir de la llegada de un "otro" deseado, como se lo consideraba al inmigrante europeo. En este contexto, de "... la escuela se esperaba mucho: en los comienzos de la Organización Nacional, se vio en ella el modo de generar unidad donde había conflictos o diferencias: entre gauchos holgazanes y citadinos cosmopolitas, entre provincianos y porteños" (Neufeld y Thisted, 1999, p. 25). Lo desarrollado hasta aquí subyacía en los contenidos de enseñanza escolar que se trataban en el contexto del aula.

El material seleccionado por la docente para trabajar la cuestión migratoria fue un texto escolar que refería al momento histórico en el que se "construyó" la nación mediante el arribo de inmigrantes provenientes de Europa. La selección de estos contenidos se producía a partir de la naturalización de la trabajadora sobre aquel ideario de ser nacional planteado en el párrafo anterior. Incluso, semanas previas, durante un recreo expresó sus dudas sobre cómo trabajar el tema. En la primera clase lo hicieron a partir de una consigna del libro de texto que proponía leer una carta enviada por un inmigrante italiano llamado Giuseppe -que contaba a sus hermanos los beneficios de haber llegado a Argentina-.

Luego de su lectura se produjo el siguiente intercambio:

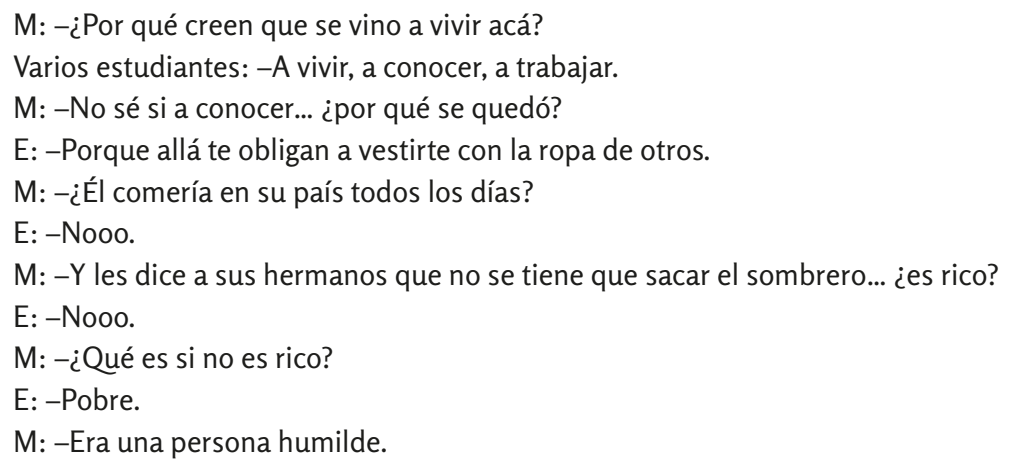


La referencia espacial que utilizó la docente para referirse al "nosotros" fue: "acá". Ello no significó tener que aclarar a qué hizo mención con ese indicativo espacial. El "acá" no era la escuela, ni la ciudad, ni la provincia. Ella se refirió al país, dejando de lado cualquier diferenciación regional que exista al interior del territorio. Luego retomó la consigna puntualizando las comparaciones que se establecían entre Italia y Argentina. En Italia Guiseppe no comía todos los días y, a través del ejemplo del sombrero, convivía al interior de un sistema social de dominación que lo obligaba a tener que sacárselo frente a los sectores poderosos. En Argentina dichas situaciones, valoradas negativamente por la docente, no ocurrían y el inmigrante podía alimentarse bien al tiempo que se liberaba de esas prácticas serviles. El relato trabajado en la clase no hizo mención, como plantea Segato (2007), al ejercicio de las instituciones de aplanar diferencias ni de las presiones a los inmigrantes a dejar de lado sus prácticas de origen (por ejemplo, la lengua), con el fin de establecer una "neutralidad étnica" para ejercer la ciudadanía. La construcción del mensaje propuesto en la consigna de una nación conformada a partir de procesos migratorios libre de tensiones y conflictos, reforzaba la consolidación de formas históricas y hegemónicas de construir al "ser nacional".

Para comprender estos procesos es importante tener presente que la explicitación de esta idea que sostiene la "neutralidad", reforzada desde diferentes instituciones y actores, no es la expresión de una política de imposición. En este caso, la docente planteó esa idea de "ser nacional" porque la reconocía como válida y legítima, estableciendo para ella un sentido de la realidad (Williams, 1977). La complejidad con que esto ocurrió supera los posicionamientos que plantean como explicación la reproducción de contenidos escolares "oficiales" enseñados obligatoriamente por docentes. En vez de ello, los contenidos del diseño curricular refieren a relatos que se vinculan con la forma en que las personas descendientes de inmigrantes reconstruyen su historia familia. En este sentido, es importante...
... no perder de vista que, en la relación nosotros-otros, la construcción de la alterización se realiza [...] en la elaboración de marcos teóricos-conceptuales y, por otro, en la carga de significados, interpretaciones y representaciones que los actores sociales les atribuyen a esas diferencias. (Sinisi, 1999, p. 205)

Así, la subjetividad de la docente al momento de enseñar estaba atravesada por una visión que legitimaba la idea de una nación homogénea y libre de conflictos. Estos procesos, al ser internalizados en la práctica y formados en el área total de experiencia de vida, incidían en la percepción, los afectos y pensamientos de los actores (Ortner, 2005). Esto ocurrió cuando la docente vinculó la consigna de la clase con algunos pasajes de la experiencia de su abuelo.

\footnotetext{
M: -Mucha gente vino de Italia porque no tenía trabajo, por la guerra... yo les cuento una historia. Mi abuelo no nació en Argentina...

E: -Nació en Alemania.

M: -Muy bien, cómo te acordás. Era muy jovencito y había guerra. Él no quería estar en la guerra y vino con los otros tres hermanos. Se fueron a Tres Arroyos... ¿conocen? [Silencio, ningún estudiante responde].

M: - ¿A qué se fue? A sembrar trigo en los campos. Mi abuelo vino en 1926. No había máquinas como ahora y los alemanes eran buenos labradores. Tuvo cuatro hijas...
}

En la configuración y generación de subjetividades tienen un papel muy importante las formaciones culturales definidas como "... el cuerpo relativamente coherente de símbolos y significados, ethos y visión del mundo...” (Ortner, 2005, p. 31). La docente, al relatar la huida de su familiar a la Argentina producto de la guerra, lo convirtió en un actor que aceptaba ser parte del proceso de construcción nacional a partir del trabajo y la 
producción, manifestando un conjunto de aspectos similares a la historia de Giuseppe mencionada en el texto escolar. En este sentido, el "... nacionalismo ha dejado hondas huellas en programas y textos escolares, en símbolos y emblemas, y también en el imaginario de muchos maestros argentinos" (Diez \& Novaro, 2011, p. 7). Ambos relatos estaban vinculados a la formación maestra de alteridad en Argentina, considerando la incorporación del progreso por el puerto y la selectividad de alteridades para la "argentinización" (Briones, 2005). Sobre esta compleja construcción de lo nacional, la docente inició la clase para abordar el tema inmigraciones refiriéndose a los "otros" desde el "nosotros" estableciendo la frontera allí donde el Estado argentino tenía soberanía.

\section{Otras fronteras: complejizando la definición unívoca de inmigrante}

Las fuerzas hegemónicas que construyen sentidos de realidad sobre el "ser nacional" y definen al inmigrante por fuera del territorio argentino no son únicas ni totales. Existen formas que se contraponen e incluso pueden resistir esa visión que se presenta como unívoca, aunque no sean necesariamente conscientes ni se estructuren en formas políticamente organizadas de resistencia. Pueden tratarse, como en este caso, de planteos realizados por niños a partir de la experiencia que realizan en su vida cotidiana. Esto se relevó en el momento en que la docente, luego de introducir al tema y plantear la consigna de Giuseppe, propuso que todos los/as estudiantes participen preguntándoles si conocían inmigrantes:

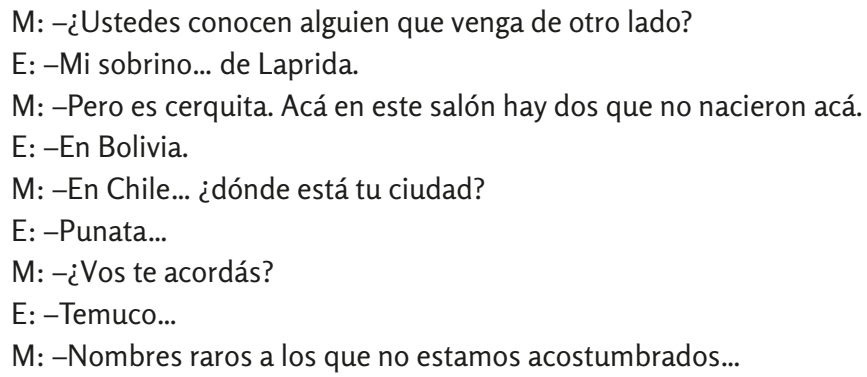

La situación se repite en otro momento de la clase:

E: -Mi mamá nació en Buenos Aires.

M: - ¿Es otro país? Yo estoy hablando de la gente que vino de otro país. Ahora abran el cuaderno y van a tener que investigar:

Anotan como título: "Vinieron de otro país". Y la consigna dice: "Averiguo en casa si hay algún familiar que nació en otro país y por qué vino."

En el primer ejemplo, nuevamente, aparece la referencia "acá" que, como se mencionó anteriormente, establecía el límite en el territorio argentino. Así, la referencia a la "otredad" en el marco de la construcción de la categoría inmigrante fue circunscripta a personas que no nacieron en Argentina. Sin embargo, para las/os niñas/os la "otredadinmigrante" se presentaba de manera más cercana discutiendo con la exclusividad de la frontera vinculada al territorio nacional. Para un niño, el otro lado de la frontera donde se encontraban los inmigrantes era una localidad como Laprida, ubicada a ciento veinte kilómetros de la ciudad. Esto generó la redefinición de la categoría frontera otorgándole mayor complejidad. Esta oposición a la definición de la categoría inmigrante planteada en el apartado anterior, vinculada a procesos de construcción sociohistórica de carácter hegemónico, fue cuestionada por la docente. Laprida estaba "cerca", era parte del país ("acá"), era parte del "nosotros"; Punata y Temuco eran desconocidas, eran nombres "raros", estaban lejos y constituían la "otredad". La situación particular de esta interacción en el aula se incorpora a formas generales en donde el... 
... trabajo docente, en general, se [ha] manejado con versiones del nacionalismo donde "los otros", los extranjeros, los indígenas, los migrantes ocupan el lugar de una diversidad objeto de miradas sospechosas, de representaciones que alternan entre la desvalorización y el exotismo. (Novaro, 2011c, p. 1)

En el ejemplo mencionado, las intervenciones de los niños no podían tener lugar sin poner en discusión la idea hegemónica sobre el "ser nacional". A pesar de los intentos de la docente, los signos de oposición a la construcción hegemónica estaban latentes y la situación se repitió nuevamente.

En el segundo ejemplo, la indicación que realiza un niño sobre los inmigrantes hizo referencia a quienes nacían en la capital de Argentina y en el lenguaje "nativo" eran denominados porteños. Esta no era una cuestión que respondía a la imaginación del niño. Existen investigaciones socioantropológicas que se refieren tanto a las fronteras físicas - la avenida Gral. Paz (Gravano, 2003)- como simbólicas - "cabecita negra" (Ratier, 1971)- existentes entre Capital Federal y el llamado "interior" (categoría en la que se agrupan el resto de las provincias). A pesar de ello, al abordar el tema no se mencionaron los procesos internos de inmigración reafirmando la idea de "otredad" migratoria por fuera de las fronteras del territorio argentino. La situación registrada mostró cómo los niños ponían de relieve la forma superficial que un país se entrama a partir de la convivencia de grupos/sociedades/pueblos con culturas tan propias y diferentes como valiosas (Briones, 2005). Esto no deja de ser válido, incluso para la docente. El problema fue que dicha diferenciación se oponía al enfoque planteado desde la idea hegemónica de "ser nacional" - expresada en los contenidos curriculares-, poniendo en duda la representación homogénea que se oponía a la construcción del inmigrante. Frente a ello la docente desestimó esa idea a partir de distintos argumentos ya que no eran acordes a los contenidos que intentaba plantear en términos nacionales. Lo que subyacía a sus planteamientos era el estado de alerta hacia alternativas que cuestionaban la dominación (Williams, 1977) de los significados históricos sobre el "ser nacional".

$\mathrm{Al}$ realizar este tipo de acercamientos analíticos es importante evitar esencializar a los actores caracterizándolos como hegemónicos o contrahegemónicos. Los seres humanos están atravesados por contradicciones que se vinculan y separan de las formaciones socioculturales hegemónicas que, en este caso, se refieren a las formaciones nacionales de alteridad. Un ejemplo de ello ocurrió en la segunda clase donde, al tratarse el tema inmigración, se invitó a una inmigrante chilena con el objetivo que relatara cuestiones vinculadas a su país de origen. En relación a la perspectiva planteada por la docente, la inmigrante -con un libro en la mano- comenzó brindando algunos datos sobre la fundación de Chile, mencionó la minería como el recurso principal del país, habló de su flora y su fauna (el copihue y el cóndor como símbolos nacionales), las formas de alimentación y los nombres de algunas comidas, sus divisiones regionales, las características de la bandera y las "danzas nacionales". Sin embargo, en su relato sobre el "ser nacional" chileno se expresaron otras formas de construcción de alteridad que estaban por fuera de las fronteras nacionales. Vinculado al relato entrelazó su experiencia de vida en el lugar de origen y, en algunas situaciones, mencionó al pueblo Mapuche que extiende su territorio -no reconocido desde el Estado- por la zona chilena y argentina. A partir de ello, el "ser nacional", visto como una construcción hegemónica, comenzó a ser redefinido. Habló del origen de los mapuches, aclaró que también hablaban español, aunque aprender su idioma no era difícil y ella lo había hecho cuando empezó a hacer artesanías (incluso mostró su conocimiento diciendo una frase en esa lengua). La docente le preguntó si era descendiente y respondió que no, pero que le "hubiese gustado". Contó que hacían pulseras de cobre que curaban el reuma, secaban el cultrún y hacían instrumentos musicales. Destacó que las mujeres mapuches tejían muy bien y hacían frazadas, mientras el hombre trabajaba la tierra. 
Fue tanto el interés que se produjo sobre su experiencia con el pueblo mapuche que relató cómo, en la zona de Temuco, cuando alguien deseaba una novia se la tenía que robar, y luego le pedían la "dote" (caballos, oro, plata) que el padre tenía que ceder. También contó que eran siete mujeres las que podía tener el cacique y que cuando fallecía una persona mapuche le hacían un ritual. De esta forma, en su relato sobre el "ser nacional" de Chile, irrumpieron destellos de experiencias que iban más allá de los límites nacionales. La ruptura que produjo este relato puede ser comprendida mediante una perspectiva transnacional. Si bien los estudios que hacen uso de esa categoría se refieren, generalmente, a las redes y vínculos que mantienen los inmigrantes con el país de origen -contra las perspectivas asimilacionistas-, aquí también resulta pertinente. En primer lugar, porque en el discurso de la inmigrante se visualizó la existencia de identidades que la ligaban con el lugar de origen. En segundo lugar, porque se refirió a la existencia de un campo social transnacional (Levitt \& Glick Schiller, 2004, p. 66) -territorio mapuche-cuyas fronteras no coinciden con los límites de los estados nacionales. Si bien reconoció que ella no se encontraba inserta en las redes e interacciones que envolvían a dicho campo, su referencia permitió mostrar la existencia de formas que irrumpieron en la construcción social de la categoría inmigrante limitada a las formaciones nacionales de alteridad.

\section{Consideraciones finales}

El relevamiento realizado en las clases donde se trabajó el tema inmigración es una muestra de la complejidad que atraviesa las formas en que se construye la idea de inmigrante. Las adscripciones categoriales planteadas por la docente para diferenciar "nativos" de inmigrantes se orientaban a la nacionalidad. El límite que los dividía asumió formas simbólicas e históricas que decantaban en las fronteras territoriales entre países. Dicha definición consolidó la existencia de un "ser nacional" homogéneo sustentado en relatos históricos. Sin embargo, esta formación es arbitraria, evidenciándose la existencia de otras formas de construir las fronteras que dividen al nativo del inmigrante y que ponen en duda su exclusividad unívoca. Si bien los actores aceptaron la definición que establecía los límites territoriales nacionales, fue posible reconocer otras definiciones que producían límites al interior o más allá de la "soberanía nacional". Dichas definiciones identificadas tenían vinculación con conflictos y tensiones que hicieron a la conformación de lo que hoy se conoce como Argentina. Algunas referían a las luchas ocurridas entre las regiones internas por la organización y administración que derivaron en lo que se conoce como territorio nacional. Otras, a la existencia de grupos como los pueblos originarios que fueron perseguidos, expulsados y exterminados en el proceso que decantó en la instauración del Estado argentino.

A pesar de que la mayoría de los actores que participaron de las situaciones registradas poseían la misma nacionalidad, el tratamiento del tema generó situaciones culturales e identitarias de gran complejidad. Las primeras se refieren a la producción de identificaciones que “... distintos en la sociedad, se relacionan y comunican produciéndose conflictos, negociaciones, acuerdos e innumerables malos entendidos" (Grimson, 1999 , p. 35). Mientras que las situaciones identitarias expresan la inexistencia de una identidad "natural" unívoca (Grimson, 1999). En este caso, se trata de niños y niñas de siete u ocho años que produjeron otras distinciones, además de las definidas desde la institución escolar, siendo el relato de la "historia oficial" una entre otras alternativas. La importancia de objetivar estos procesos radica en que permiten complejizar la construcción social de la categoría inmigrante, entendiéndola como un producto histórico, político y social atravesado por situaciones de tensión y conflicto entre diferentes sectores. Esto permite reconocer las características de las fronteras que producimos y 
reproducimos como instancias de diferenciación, así como reflexionar sobre nuestro lugar en la sociedad -en términos de extrañamiento- siendo que, además de pertenecer a un "nosotros", también formamos parte de un "otro".

Llevar adelante estas conceptualizaciones permitiría un principio de trabajo que evite esencializar a los inmigrantes y sustancializar las categorías sociales elegidas para elaborar su construcción. Esta perspectiva plantea la necesidad de profundizar la investigación incluyendo la posibilidad de vincular el enfoque socioantropológico a las prácticas de la comunidad educativa. En este sentido, es posible pensar iniciativas que se orienten a reflexionar cómo los actores que conforman la institución representan la inmigración y qué conocimientos tienen sobre dicho fenómeno. De esta manera, es también necesario analizar el material bibliográfico con el que cuentan los docentes para el tratamiento del tema, generando la posibilidad de reflexionar sobre los mismos con el objetivo de que los actores elaboren contenidos actualizados que se vinculen a sus contextos. Todo esto, teniendo como meta que las personas puedan reconocerse como "otros" mediante la visualización de las diferencias y desigualdades que organizan a la sociedad.

Lo planteado hasta aquí constituye las primeras consideraciones en el tratamiento sobre las producciones de alteridad que ocurren en contextos escolares. Sin duda, queda mucho por continuar problematizando reconociendo las múltiples aristas a las que refiere el tema. En este marco, resultan pertinentes algunas consideraciones que expresan referentes del campo subdisciplinar de la Antropología y Educación a fin de lograr lo planteado. En principio, teniendo en cuenta que ante las distintas formas que asume el nacionalismo, “... los niños se posicionan de diferente manera frente a estas interpelaciones, sus referencias identitarias se complejizan y frecuentemente algunas de estas referencias entran en tensión los mandatos y discursos educativos" (Novaro, 2011 b, p. 179). Analizando esto, resulta necesaria “... la discusión y negociación de significados desde un currículum escolar que reafirma un 'modo instruccional dominante' de transmisión de información y confianza acrítica en los contenidos..." (Díaz, Diez \& Thisted, 2009, p. 27). Principalmente, como plantean los trabajos citados, docentes y estudiantes creen y confían en los libros de texto como fuente de conocimiento. Y esto constituye un ejemplo de cómo "... los Estados, a través de distintas políticas educativas, sociales y culturales, promueven algunas identidades y niegan o limitan otras" (Díaz, Diez \& Thisted, 2009 citado por Diez, 2011, p. 155). Por ello, resulta necesario continuar indagando en torno a estas cuestiones. 


\section{- Referencias bibliográficas}

» Barth, F. (1976). Los grupos étnicos y sus fronteras. México: Fondo de Cultura Económica.

"Briones, C. (2005). Formaciones de alteridad: contextos globales, procesos nacionales y provinciales. En Briones, C. (ed.), Cartografías argentinas, pp. 9-36. Buenos Aires: Antropofagia.

»Díaz, R., Diez, M. L. \& Thisted, S. (2009). Educación e Igualdad. La cuestión de la educación intercultural y los pueblos indígenas. Sao Paulo: Campaña Latinoamericana por el Derecho a la Educación.

»Diez, M. L. (2011). Biografías no autorizadas en el espacio escolar. Reflexiones en torno a ser migrante en la escuela. En Novaro, G. (coord.), La interculturalidad en debate. Experiencias formativas y procesos de identificación de niños indígenas y migrantes. Buenos Aires: Biblos.

»Diez, M. L. \& Novaro, G. (2011). ¿Una inclusión silenciosa o las sutiles formas de discriminación? En Courtis, C. \& Pacceca M. I. (comps.), Discriminaciones étnicas y nacionales. Un diágnostico participativo. Buenos Aires: Del Puerto y Asociación por los Derechos Civiles.

»El Popular (31 de marzo de 2014). El 1,5\% de la población en Olavarría es extranjera. Recuperado de: http://www.elpopular.com.ar/eimpresa/184501/el15-de-la-poblacion-deolavarria-es-extranjera

» Feldman-Bianco, B., Rivera Sánchez, L., Stefoni, C. \& Villa Martínez, M. (2010). Introducción. En Feldman-Biando, B., Rivera Sánchez, L., Stefoni, C., Villa Martínez, M. (comp.), La construcción social del sujeto inmigrante en América Latina: prácticas, representaciones y categorías, pp. 15-30. Buenos Aires: CLACSO - FLACSO.

» Franzé Mudanó, A. (2002). Lo que sabía no valía. Escuela, diversidad e inmigración. Madrid: Consejo Económico y social, Comunidad de Madrid.

"Gravano, A. (2003). Los atrases y delantes de las ciudades, muestra del trabajo con los imaginarios urbanos. Runa. Archivos de las Ciencias del Hombre, 24(1), 27-42.

»_. (2006). Imaginarios regionales y circularidad en la planificación: el caso del TOAR. Intersecciones en Antropología, (7), pp. 305-323.

" Grimson, A. (1999). Relatos de la diferencia y la igualdad. Los bolivianos en Buenos Aires. Buenos Aires: Eudeba.

"Levinson, B. \& Holland, D. (1996). The Cultural Production of the Educated Person: An Introduction. En Levinson B., Foley, D. \& Holland, D. (comps.), The cultural production of the educated person, pp. 1-56. Albany: State University of New York.

" Levitt, P. \& Glick Schiller, N. (2004). Perspectivas internacionales sobre migración: conceptualizar la simultaneidad. Migración y Desarrollo, (3), 60-91.

" Neufeld, M. R. (2009). Antropología y educación en el contexto argentino. VIII Reunión de Antropología del MERCOSUR. Buenos Aires: Universidad Nacional de San Martín. 
» Neufeld, M. R. \& Thisted, A. (1999). El crisol de razas hecho triza. En Neufeld, M. R. y Thisted, A. (comps.), De eso no se habla... los usos de la diversidad sociocultural en la escuela. Buenos Aires: Eudeba.

» Novaro, G. (2011a). Introducción. Interculturalidad y Educación. Reflexiones desde las experiencias formativas de niños indígenas y migrantes. En Novaro, G. (coord.), La interculturalidad en debate. Experiencias formativas y procesos de identificación de niños indígenas y migrantes. Buenos Aires: Biblos.

$» \ldots$ _. (2011b). Niños migrantes y escuela: ¿identidades y saberes en disputa? En Novaro, G. (coord.), La interculturalidad en debate. Experiencias formativas y procesos de identificación de niños indígenas y migrantes. Buenos Aires: Biblos.

> (2011C). Nacionalismo escolar y experiencias formativas de migrantes bolivianos en Buenos Aires. Entre el reconocimiento, la invisibilización y el silenciamiento. Boletín de Antropología y Educación, (2). Recuperado de: http:// antropologia.institutos.filo.uba.ar/sites/antropologia.institutos.filo.uba.ar/ files/bae_nozao3.pdf

"Ortner, S. (2005). Geertz, subjetividad y conciencia posmoderna. Etnografías contemporáneas, (1), 25-47.

» Ratier, H. (1971). El cabecita negra. Buenos Aires: Centro Editor de América Latina.

"Rockwell, E. (2009). La experiencia etnográfica. Historia y cultura en los procesos educativos. Buenos Aires: Paidós.

"Segato, R. (2007). La Nación y sus otros. Raza, etnicidad y diversidad religiosa en tiempos de Política de la Identidad. Buenos Aires: Prometeo.

" Sinisi, L. (1999). La relación nosotros-otros en espacios escolares "multiculturales". Estigma, estereotipo y racialización. En Neufeld, M. R. y Thisted, A. (comps.), De eso no se habla... los usos de la diversidad sociocultural en la escuela. Buenos Aires: Eudeba.

"Tevez, E. (2011). La experiencia migratoria en el marco de la Ley 25871. Trabajo presentado en X Congreso Nacional de Antropología Social. Facultad de Filosofía y Letras. Universidad de Buenos Aires. Buenos Aires, Argentina.

»Williams, R. (1977). Marxismo y Literatura. Buenos Aires: Manantial.

\section{Bibliografía de consulta}

»Briones, C. (2008). Diversidad cultural e interculturalidad: ¿de qué estamos hablando? En García Vázquez, C. (comp.), Hegemonía e interculturalidad, Poblaciones originarias e ininmigrantes, pp. 35-58. Buenos Aires: Prometeo.

» Novaro, G. (2009). Palabras desoídas - palabras silenciadas - palabras traducidas. Voces y silencios de niños bolivianos en escuelas de Buenos Aires. En Dossiê Antropologia da Educação. Centro de Educação, 34. Laboratório de Pesquisa e Documentação - LAPEDOC. Universidade Federal de Santa Maria. 


\section{Emilio Tevez}

Doctor en Antropología, Universidad de Buenos Aires. Becario posdoctoral del Consejo Nacional de Investigaciones Científicas y Técnicas, Facultad de Ciencias Sociales, Universidad Nacional del Centro de la Provincia de Buenos Aires, Olavarría, Argentina. Correo electrónico: tevezemilio@gmail.com

\section{Sofía Dueñas Díaz}

Tesista de grado, Licenciatura en Antropología Social, Facultad de Ciencias Sociales, Universidad Nacional del Centro de la Provincia de Buenos Aires, Olavarría, Argentina. Correo electrónico: duediazsofia@gmail.com 